RESEARCH

\title{
Prealbumin to fibrinogen ratio is closely associated with diabetic peripheral neuropathy
}

\author{
Shufei Zang ${ }^{1}$, Lei Shi' ${ }^{2}$, Jinying Zhao ${ }^{1}$, Min Yang ${ }^{1}$, Jun Liu ${ }^{1}$ and Heyuan Ding ${ }^{1}$ \\ 1'Department of Endocrinology, Shanghai Fifth People's Hospital, Fudan University, Shanghai, China \\ 2Department of Neurology, Shanghai Fifth People's Hospital, Fudan University, Shanghai, China
}

Correspondence should be addressed to H Ding: heyuan.ding@fudan.edu.cn

\begin{abstract}
The aim of our study was to explore the diagnostic value of prealbumin to fibrinogen ratio (PFR) for predicting prognosis with the optimal cut-off value in diabetic peripheral neuropathy (DPN) patients. A total of 568 type 2 diabetes mellitus (T2DM) patients were enrolled in this study. The values including Toronto clinical neuropathy score (TCNS), nerve conduction velocity (NCV), vibration perception threshold (VPT), blood cells count, biochemical parameters, fibrinogen and PFR were recorded. The patients were divided into tertiles based on admission PFR value. First, clinical parameters were compared among the groups. Secondly, a logistic regression and ROC analysis were performed as the statistical model. The percentage of DPN, TCNS and VPT were significantly higher in the lowest PFR tertile than in the middle PFR tertile and the highest PFR tertile $(P<0.01-0.001)$. NCV was significantly lower in lowest PFR tertile than in the middle PFR tertile and the highest PFR tertile $(P<0.01-0.001)$. The Spearman correlation analysis showed that PFR was negatively correlated with TCNS and VPT $(P<0.001)$, while PFR was positively correlated with median motor NCV $(P<0.001)$, peroneal motor NCV $(P<0.001)$, median sensory NCV $(P<0.001)$, and peroneal sensory NCV $(P<0.001)$. After adjusting these potentially related factors, PFR was independently related to DPN $(P=0.007)$. The area under ROC curve was 0.627 . This study finds the first evidence to suggest PFR may be the key component associated with DPN in T2DM, while PFR might underlie the pathophysiologic features of DPN.
\end{abstract}

\author{
Key Words \\ - diabetes mellitus \\ - type 2 \\ - prealbumin to fibrinogen \\ ratio \\ - diabetic peripheral \\ neuropathy \\ - vibrating perception \\ threshold \\ - nerve conduction velocity
}

\section{Introduction}

Type 2 diabetes mellitus (T2DM) is a chronic metabolic disease, characterized by the hyperglycemia level and insulin resistance in the body. T2DM is also considered to be induced by personal lifestyle, such as high consumption of carbohydrates, and lack of physical exercises. The increasing prevalence of T2DM worldwide is a major global public health burden $(1,2$, $3)$. According to the International Diabetes Federation, there are currently 366 million diabetes mellitus patients worldwide and it is expected to increase to 522 million by 2030 (4). Diabetic peripheral neuropathy (DPN) is one of the most complicated pathological changes occurring in T2DM with an incidence of about 50\%. The status of DPN may also lead to higher morbidity and mortality, which increases the financial burden of T2DM treatment $(5,6)$. Furthermore, DPN is responsible for the development of various serious diseases, such as foot ulcer, infection, gangrene and non-traumatic lower limb amputation. These complications seriously affect the patient's quality of life $(7,8)$. DPN has been considered as an inflammatory disease. However, its pathogenesis in T2DM has not been fully elucidated $(9,10,11,12)$.

Fibrinogen (FIB) is a biomarker of coagulation and chronic inflammation (13). And a high FIB level is correlated with systemic inflammation (14). Impairment of FIB level is also associated with microvascular disease

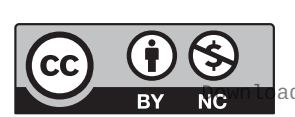

This work is licensed under a Creative Commons Attribution-NonCommercial 4.0 International License. ded from Bioscientifica.com at 04/26/2023 12:08:01PM 
in patients with T2DM (15). Albumin (ALB) is a nutrition marker and an inflammation marker (16). Li et al. showed the serum ALB was independently associated with peripheral nerve function in T2DM patients, especially in those with albuminuria (17). In recent studies, the prealbumin (PALB) is served as another important biomarker for nutritional status. It is more sensitive to malnutrition than $\operatorname{ALB}(18,19)$. In addition, previous studies have confirmed that PALB values are also inversely related to CRP values in inflammation $(20,21,22)$. PALB to FIB ratio (PFR) is a new inflammation marker, which is closely related to acute pancreatitis and cancer $(23,24)$. Therefore, we speculate that PFR may also be associated with DPN in T2DM.

PALB and FIB are useful tools in various research because they are cheap and easy to use. However, no study has assessed the prognostic role of PFR in DPN patients so far. The aim of our study was to explore the diagnostic value of PFR for predicting prognosis with the optimal cut-off value in DPN patients.

\section{Materials and methods}

\section{Study design and patients}

The study was carried out from January 2018 to December 2019. In total, 568 T2DM patients were recruited from inpatient department of endocrinology of Shanghai Fifth People's Hospital, Fudan University. The diagnostic criteria of T2DM were referred to American Diabetes Association standards (25). Patients with alcohol abuse, vitamin deficiency, liver dysfunction (alanine aminotransferase (ALT) greater than 2.5 times the normal upper limit), renal dysfunction (estimated glomerular filtration rate (eGFR) less than $60 \mathrm{~mL} / \mathrm{min} / 1.73 \mathrm{~m}^{2}$ ), acute cerebral infarction, amyotrophic lateral sclerosis, Alzheimer disease, Parkinson disease, and other disorders of the CNS were excluded from this study. This study was conducted under the program of risk factors of DPN and approved by the ethics committee of Shanghai Fifth People's Hospital, Fudan University (No. 2018-213). Consent has been obtained from each patient or subject after full explanation of the purpose and nature of all procedures used.

\section{Data collection and laboratory assessments}

The patients' age and their medical history, age, duration, hypertension (HTN), BMI, systolic blood pressure (SBP), and diastolic blood pressure (DBP) were recorded.
Toronto clinical neuropathy score (TCNS) was also accessed and documented.

After a 12-h overnight fast, the patient's blood samples were collected for the measurements of hemoglobin A1c (HbA1c, Variant II, Bio-Rad), blood cells count (Automatic Blood Cell Analyzer, Sysmex XN9000), biochemical parameters test (Automatic Biochemical Analyzer, Roche Cobas 8000), and fibrinogen (FIB, CS5100, Sysmex Corporation) respectively. Neutrophil to lymphocyte ratio (NLR) is the ratio of neutrophil $\left(10^{9} / \mathrm{L}\right)$ to lymphocyte $\left(10^{9} / \mathrm{L}\right)$. Albumin to fibrinogen ratio (AFR) is the ratio of ALB $(\mathrm{g} / \mathrm{L})$ to FIB $(\mathrm{g} / \mathrm{L})$. PFR $(\mathrm{mg} / \mathrm{g})$ is the ratio of PALB $(\mathrm{mg} / \mathrm{L})$ to FIB $(\mathrm{g} / \mathrm{L})$.

\section{Nerve conduction velocity and vibration perception threshold measurement}

Peripheral nerve function is evaluated by measuring motor and sensory nerve conduction velocity (NCV). NCV was performed by a single neurologist. All nerve stimulations, including median motor nerve (MMN), peroneal motor nerve (PMN), median sensory nerve (MSN), and peroneal sensory nerve (PSN) in both limbs, were performed with an electromyography (EMG) machine (Keypoint 9033A07, Dantec Co). The local skin temperature was maintained at $32-33^{\circ} \mathrm{C}$. The variables were considered abnormal when they exceeded mean \pm 2 s.D. that were established in the authors' laboratory. Vibration perception threshold (VPT) was measured by a trained nurse using a digital vibration threshold detector (Sensitometer A200, Beijing Blue Time's Technology Co).

\section{DPN diagnosis}

DPN was diagnosed according to Toronto Expert Consensus (26) as follows: clear history of diabetes; peripheral neuropathy occurring at or after diagnosis of diabetes; clinical symptoms and signs consistent with performance of DPN; a neurologic symptom or symptoms (foot pain, numbness, tingling, weakness, ataxia, or upper-limb symptoms, etc.); a neurologic sign or signs (acupuncture pain, touch pressure, temperature, vibration, or ankle reflex, etc.). The presence of an abnormality of nerve conduction and a symptom or symptoms or a sign or signs of neuropathy confirm DPN.

\section{Statistical analysis}

All statistical analyses were performed using the Statistical Package for Social Sciences (SPSS) Version 22.0.

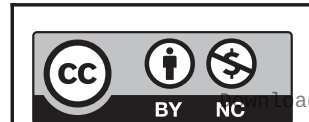

This work is licensed under a Creative Commons Attribution-NonCommercial 4.0 International License. ded from Bioscientifica.com at 04/26/2023 12:08:01PM via free access 
Normally distributed continuous variables were expressed as means \pm S.D. and analyzed by using Student's $t$-test. Non-normally distributed variables were expressed as median and interquartile range (IQR), and analyzed by using nonparametric test (Wilcoxon test). The categorical variables were presented as frequencies and proportions, and analyzed by using $\chi^{2}$ test. Furthermore, the Spearman correlation analysis was performed to evaluate the association of the parameters of DPN. The binary logistic regression analysis was performed to evaluate the association of PFR and DPN after adjusting other clinical and biochemical variables. $P$ values of less than 0.05 were regarded as statistically significant.

\section{Results}

\section{Demographics of the study population}

The clinical characteristics of the study population were shown in Table 1. Compared with non-DPN group, age $(P<0.001)$, duration $(P<0.001)$, HTN $(P=0.039)$, TCNS $(P<0.001)$, creatinine (Crea, $P=0.008)$ and NLR $(P<0.001)$ of DPN group were significantly increased, while fasting plasma glucose (FPG, $P=0.041$ ), total cholesterol (TC, $P=0.027)$, AFR $(P<0.001)$ and PFR $(P<0.001)$ were significantly decreased. There were no significant differences in BMI, systolic blood pressure (SBP), diastolic blood pressure (DBP), HbA1c, ALT, urea nitrogen (UN), uric acid (UA), triglyceride (TG), HDL-C, and highly sensitive $\mathrm{C}$-reactive protein (hs-CRP) between the two groups.

Interestingly, PFR was significantly lower in patients with DPN (Table $1, P<0.001$ ). Patients were divided into tertile groups based on PFR (the lowest tertile $<80.85$, the middle tertile $80.85-114.85$, the highest tertile $\geq 114.85$ ). In the lowest, middle and highest tertile PFR groups, the percentages of DPN were 58.2, 39.5 and $33.3 \%$, respectively (Fig. 1A). The percentage of DPN was significantly higher in the lowest tertile than in the middle tertile and the highest tertile (Fig. $1 \mathrm{~A}, P<0.001$ ). We also analyzed the differences between TCNS, VPT and NCV among the tertile groups. TCNS and VPT were significantly higher in the lowest tertile than in the middle tertile and the highest tertile (Fig. $1 \mathrm{~B}$ and $\mathrm{C}, P<0.01-0.001$ ). NCV was significantly lower in the lowest tertile than in the middle tertile and the highest tertile (Fig. 1D, E, F and G, $P<0.01-0.001)$.

Table 1 Characteristics of the study population.

\begin{tabular}{l}
\hline Variables \\
\hline N (male/female) \\
Age (years) \\
Duration (years) \\
HTN, $n(\%)$ \\
BMI $\left(\mathrm{kg} / \mathrm{m}^{2}\right)$ \\
SBP $(\mathrm{mmHg})$ \\
DBP $(\mathrm{mmHg})$ \\
TCNS \\
HbA1c $(\%)$ \\
FPG $(\mathrm{mmol} / \mathrm{L})$ \\
ALT $(\mathrm{U} / \mathrm{L})$ \\
Crea $(\mu \mathrm{mol} / \mathrm{L})$ \\
UN $(\mathrm{mmol} / \mathrm{L})$ \\
UA $(\mathrm{mmol} / \mathrm{L})$ \\
TC $(\mathrm{mmol} / \mathrm{L})$ \\
TG $(\mathrm{mmol} / \mathrm{L})$ \\
HDL-C $(\mathrm{mmol} / \mathrm{L})$ \\
hs-CRP $(\mathrm{mg} / \mathrm{L})$ \\
NLR \\
AFR \\
PFR (mg/g)
\end{tabular}

\begin{tabular}{c}
\hline Total \\
\hline $568(263: 305)$ \\
$60.3 \pm 11.5$ \\
$9.0 \pm 7.2$ \\
$336(59.2)$ \\
$24.8 \pm 3.7$ \\
$131 \pm 18$ \\
$78 \pm 10$ \\
$5(1,9)$ \\
$9.3 \pm 2.1$ \\
$8.7 \pm 3.4$ \\
$25.7 \pm 25.7$ \\
$63.3 \pm 15.6$ \\
$5.25 \pm 1.49$ \\
$292 \pm 89$ \\
$4.67 \pm 1.27$ \\
$1.91 \pm 2.34$ \\
$1.15 \pm 0.36$ \\
$3.6 \pm 5.4$ \\
$2.37 \pm 1.68$ \\
$16.3 \pm 4.7$ \\
$99.1 \pm 37.6$
\end{tabular}

\begin{tabular}{c}
\hline Non-DPN \\
\hline $320(144: 176)$ \\
$56.4 \pm 11.9$ \\
$7.2 \pm 6.6$ \\
$177(55.3)$ \\
$25.1 \pm 4.0$ \\
$131 \pm 18$ \\
$79 \pm 10$ \\
$2(0,3)$ \\
$9.3 \pm 2.2$ \\
$8.9 \pm 3.4$ \\
$26.0 \pm 19.8$ \\
$61.8 \pm 14.9$ \\
$5.17 \pm 1.42$ \\
$294 \pm 92$ \\
$4.78 \pm 1.28$ \\
$1.97 \pm 2.33$ \\
$1.15 \pm 0.34$ \\
$3.5 \pm 5.0$ \\
$2.15 \pm 1.11$ \\
$17.1 \pm 4.5$ \\
$106.5 \pm 38.4$
\end{tabular}

\begin{tabular}{c}
\hline DPN \\
\hline $248(119: 129)$ \\
$65.3 \pm 8.6$ \\
$11.2 \pm 7.3$ \\
$159(64.1)$ \\
$24.5 \pm 3.2$ \\
$132 \pm 18$ \\
$77 \pm 10$ \\
$10(7,12)$ \\
$9.3 \pm 2.0$ \\
$8.3 \pm 3.3$ \\
$25.2 \pm 31.7$ \\
$65.3 \pm 16.2$ \\
$5.35 \pm 1.58$ \\
$288 \pm 84.8$ \\
$4.54 \pm 1.24$ \\
$1.84 \pm 2.37$ \\
$1.16 \pm 0.38$ \\
$3.7 \pm 5.9$ \\
$2.64 \pm 2.17$ \\
$15.3 \pm 4.8$ \\
$89.5 \pm 34.4$
\end{tabular}

\begin{tabular}{r}
\hline P-value \\
\hline 0.498 \\
$<0.001$ \\
$<0.001$ \\
$\mathbf{0 . 0 3 9}$ \\
0.054 \\
0.666 \\
0.074 \\
$<\mathbf{0 . 0 0 1}$ \\
0.891 \\
$\mathbf{0 . 0 4 1}$ \\
0.727 \\
$\mathbf{0 . 0 0 8}$ \\
0.142 \\
0.399 \\
$\mathbf{0 . 0 2 7}$ \\
0.535 \\
0.596 \\
0.735 \\
$\mathbf{0 . 0 0 1}$ \\
$<\mathbf{0 . 0 0 1}$ \\
$<\mathbf{0 . 0 0 1}$
\end{tabular}

Data are presented as means \pm S.D. Data of normal distribution were expressed as means \pm S.D., and analyzed by using Student's $t$-test. Data of nonnormal distribution was expressed as median and interquartile range (IQR), and analyzed by using nonparametric test (Wilcoxon test). The categorical variables were expressed as frequencies and proportions, and analyzed by using $\chi^{2}$ test. Bold indicates statistical significance ( $P<0.05$ ). AFR, albumin to fibrinogen ratio; ALT, alanine aminotransferase; BMI, BMI; Crea, creatinine; DBP, diastolic blood pressure; FPG, fasting plasma glucose; HbA1c, hemoglobin A1c; HDL-C, High density lipoprotein cholesterol; hs-CRP, highly sensitive C-reactive protein; HTN, hypertension; NLR, neutrophil to lymphocyte ratio; PFR, prealbumin to fibrinogen ratio; SBP, systolic blood pressure; TC, total cholesterol; TCNS, Toronto clinical neuropathy score; TG, triacylglycerol; UA, uric acid; UN, urea nitrogen.

https://ec.bioscientifica.com

https://doi.org/10.1530/EC-20-0316 (c) 2020 The authors Published by Bioscientifica Ltd

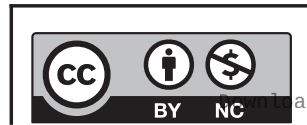

This work is licensed under a Creative Commons Attribution-NonCommercial 4.0 International License. ded from Bioscientifica.com at 04/26/2023 12:08:01PM 


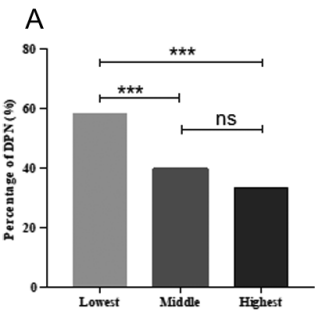

B
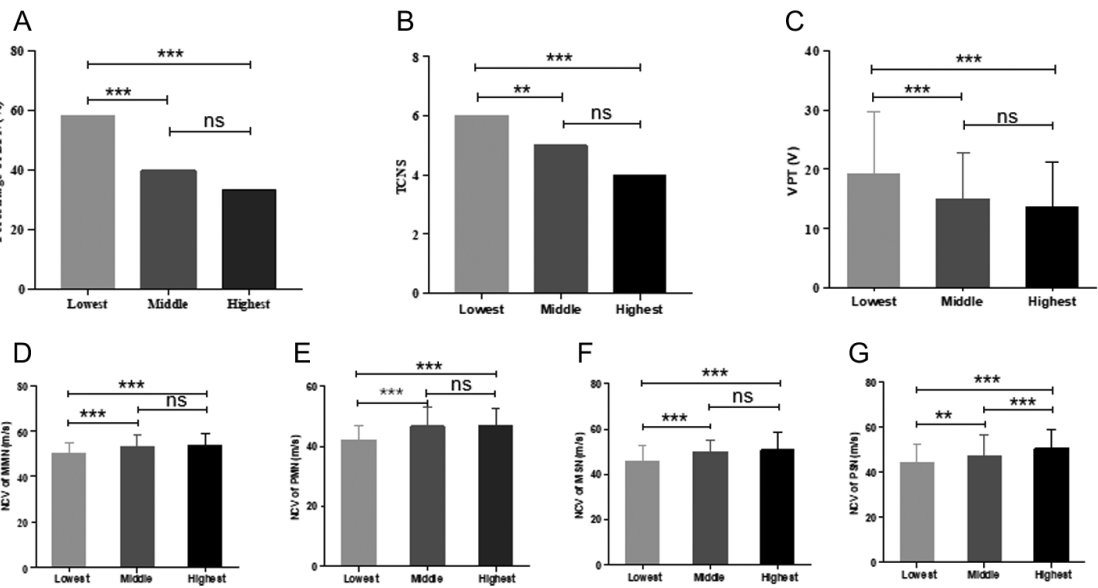

\section{Association between PFR and DPN}

Spearman correlation analysis showed that PFR was negatively correlated with TCNS and VPT (Table 2), while PFR was positively correlated with median motor NCV $(P<0.001)$, peroneal motor NCV $(P<0.001)$, median sensory NCV $(P<0.001)$, and peroneal sensory NCV $(P<0.001$, Table 2$)$.

\section{Binary logistic regression analysis}

The relationship between FPR and DPN was evaluated by using logistic regression analysis (enter method). To this end, five models were fitted: Model 1 only included PFR $(P<0.001)$; Model 2 adds age, duration and HTN to the predictors of Model $1(P=0.004)$; Model 3 added FPG and TC to the predictors of Model $2(P=0.006)$; Model 4 added Crea to the predictors of Model $3(P=0.003)$; Model 5 added NLR and AFR to the predictors of Model $4(P=0.007)$. After adjusting these potentially related factors, PFR was independently related to DPN (Table 3).

Table 2 Association of PFR with parameters of TCNS, VPT and NCV.

\begin{tabular}{|c|c|c|}
\hline & \multicolumn{2}{|c|}{ PFR } \\
\hline & rs & $P$-value \\
\hline TCNS & -0.183 & $<0.001$ \\
\hline VPT & -0.245 & $<0.001$ \\
\hline MMN-NCV & 0.239 & $<0.001$ \\
\hline PMN-NCV & 0.313 & $<0.001$ \\
\hline MSN-NCV & 0.274 & $<0.001$ \\
\hline PSN-NCV & 0.291 & $<0.001$ \\
\hline
\end{tabular}

The Spearman correlation analysis was used to evaluate the association of PFR with parameters of TCNS, VPT and NCV. Bold indicates statistical significance $(P<0.05)$

MMN, median motor nerve; MSN, median sensory nerve; NCV, nerve conduction velocity; PFR, prealbumin to fibrinogen ratio; $\mathrm{PMN}$, peroneal motor nerve; PSN, peroneal sensory nerve; TCNS, Toronto clinical neuropathy score; VPT, vibration perception threshold.

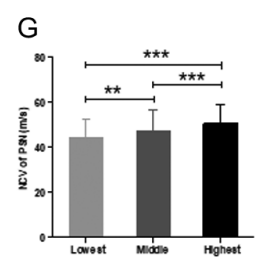

\begin{abstract}
Figure 1
Percentage of DPN, TCNS, VPT and NCV in the different PFR tertile groups. Percentages of DPN, VPT and NCV in the different PFR tertile groups. (A) Percentages of DPN in the different PFR tertile groups were analyzed by $\chi^{2}$ test. (B) TCNS, (C) VPT, (D) NCV of MMN, (E) NCV of PMN, (F) NCV of MSN, (G) NCV of PSN in the different PFR tertile groups were, respectively, analyzed by one-way ANOVA test. DPN, diabetic peripheral neuropathy; TCNS, Toronto clinical neuropathy score; VPT, vibration perception threshold; NCV, nerve conduction velocity; MMN, median motor nerve; PMN, peroneal motor nerve; MSN, median sensory nerve; PSN, peroneal sensory nerve. ${ }^{\star} * P<0.01$, $* * * P<0.001$.
\end{abstract}

\section{Sensitivity, specificity analysis and ROC}

The influence of PFR on the diagnosis of DPN was analyzed by ROC curve (Fig. 2). Area under ROC curve was $0.627(P=0.001)$. The cut-off with the biggest Yonden index of PFR was $83.31 \mathrm{mg} / \mathrm{g}$ with the sensitivity of $74.4 \%$ and specificity of $48.8 \%$.

\section{Discussion}

The inflammation, coagulation and nutrition are associated with DPN occurrence and development $(9,10$, $11,12,15,17)$. The present study finds the first evidence in suggesting that PFR is closely related with DPN in T2DM. We innovatively analyzed the relationship between PFR and DPN. From this study, the major finding we observed is that PFR is negatively associated with DPN, so it would be used as a predictor for DPN diagnosis.

In this study, we use a novel approach to demonstrate the relationship between PFR and DPN. First, FIB is a protein

Table 3 PFR associated with the presence of DPN in logistic regression (enter method).

\begin{tabular}{|c|c|c|c|}
\hline & \multicolumn{3}{|c|}{ PFR } \\
\hline & $\beta$ (S.E.) & OR $(95 \% \mathrm{Cl})$ & $P$-value \\
\hline M1 & $-0.517(0.107)$ & $0.596(0.483-0.736)$ & $<0.001$ \\
\hline M2 & $-0.336(0.117)$ & $0.715(0.568-0.899)$ & 0.004 \\
\hline M3 & $-0.328(0.120)$ & $0.720(0.570-0.911)$ & 0.006 \\
\hline M4 & $-0.359(0.121)$ & $0.698(0.550-0.886)$ & 0.003 \\
\hline M5 & $-0.478(0.178)$ & $0.620(0.437-0.879)$ & 0.007 \\
\hline
\end{tabular}

Data are presented as regression coefficient (standard error), odds ratio $(95 \% \mathrm{Cl})$ and $P$-value. Logistic regression analysis (enter method) was used to evaluate the association of PFR and DPN after adjusting other clinical and biochemical variables. Bold indicates statistical significance $(P<0.05)$. $\mathrm{M} 1$ is a regression model including just PFR; $\mathrm{M} 2$ adds age, duration and HTN to the predictors of M1; M3 adds FPG and TC to the predictors of M2; M4 adds Crea to the predictors of M3; M5 adds NLR and AFR to the predictors of M4.

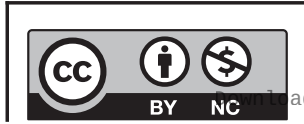

This work is licensed under a Creative Commons Attribution-NonCommercial 4.0 International License. ded from Bioscientifica.com at 04/26/2023 12:08:01PM 


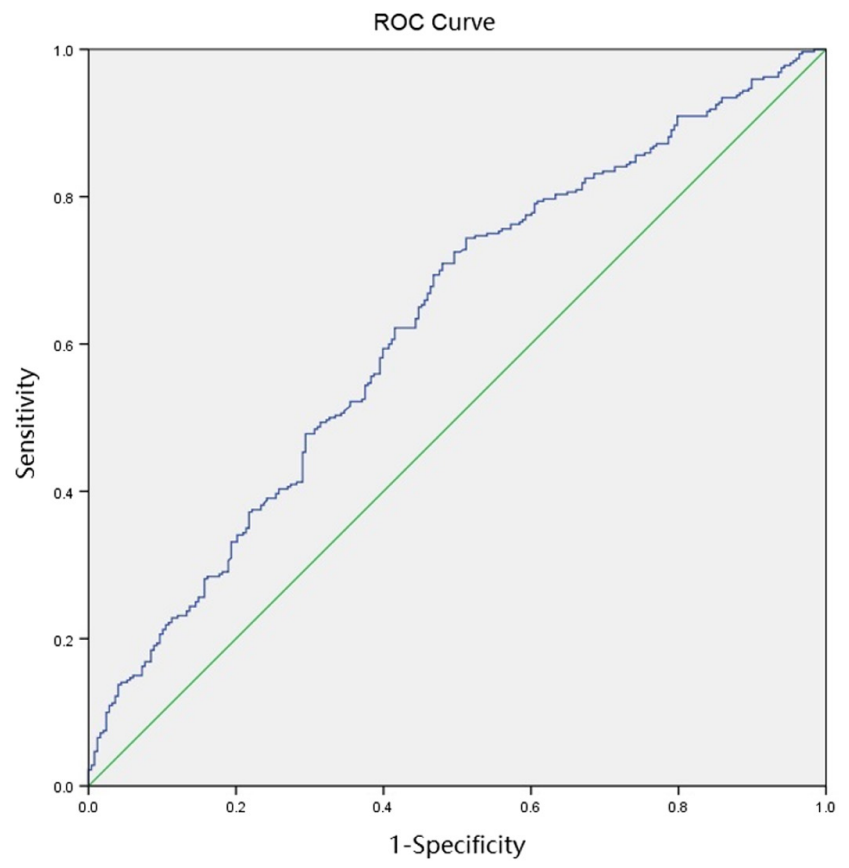

Figure 2

ROC curve of PFR for diagnosing DPN. AUC $=0.627(P<0.001)$. PFR, prealbumin to fibrinogen ratio; DPN, diabetic peripheral neuropathy; AUC, area under ROC curve.

involved in platelet aggregation and blood coagulation which is the risk factor for vascular events (27). Kotbi et al. defined that the plasma FIB concentration in Moroccan population was significantly correlated with the coronary heart disease severity (28). Besides, the elevated serum levels of FIB were also associated with diabetic end-stage renal disease in T2DM patients (29). In addition, FIB is a biomarker for chronic inflammation, as a high level of FIB is observed in systemic inflammation $(13,14)$. Secondly, ALB is an important nutritional biomarker as well as an inflammatory marker (16). Li et al. suggested that serum ALB was independent with the peripheral nerve function in T2DM, especially to those T2DM patient albuminuria (17). In addition, recent studies have reported that PALB is served as another important biomarker for nutritional status and it is more sensitive to malnutrition than ALB (18, 19). Combining two indicators of opposite changes, PFR is a valuable marker in systemic inflammatory diseases. It was reported that PFR was illustrated as the novel indicator for the diagnosis in acute pancreatitis and cancer prognosis $(23,24)$. Our study confirmed our hypothesis that PFR was associated with TCNS, VPT and NCV. Therefore, PFR can be a predicator for the diagnosis of DPN.

Although NLR, CRP and AFR are classic inflammatory indicators, studies have suggested that NLR and CRP are associated with the incidence of DPN $(9,30)$.
In our study, the differences among NLR, CRP, AFR and DPN were monitored separately. In the DPN group, NLR increased significantly, while AFR and PFR decreased significantly. Our research results partially supported other previous research results. After adjusting NLR and AFR, our regression analysis found that PFR was still an independent risk factor for DPN.

On the other hand, this study has experienced some limitations. For example, the cross-sectional method has limited us to explore the causal relationship between PFR and DPN. In future, longitudinal studies may provide better information on these relationships.

\section{Conclusions}

This study finds the first evidence to suggest PFR may be the key component associated with DPN in T2DM, while PFR might underlie the pathophysiologic features of DPN.

\section{Declaration of interest}

The authors declare that there is no conflict of interest that could be perceived as prejudicing the impartiality of the research reported.

\section{Funding}

This study was supported by Science Foundation of Shanghai Fifth People's Hospital, Fudan University (No. 2018WYZT03), Health Profession Clinical Foundation of Shanghai Municipal Health Commission (No. 201840119), and Medical Key Faculty Foundation of Shanghai (No. ZK2019B15).

\section{Author contribution statement}

Heyuan Ding designed the research. Lei Shi and Jinying Zhao performed the experiments. Min Yang analyzed the data. Shufei Zang wrote the manuscript. Jun Liu revised the manuscript. All authors read and approved the final manuscript.

\section{Acknowledgements}

The authors appreciate the time and effort contributed by all participants.

\section{References}

1 Spiller S, Frolov A \& Hoffmann R. Quantification of specific glycation sites in humanserum albumin as prospective type 2 diabetes mellitus biomarkers. Protein and Peptide Letters 201724 887-896. (https://doi. org/10.2174/0929866524666170202124120)

2 Kubisz P, Stančiaková L, Staško J, Galajda P \& Mokáň M. Endothelial and platelet markers in diabetes mellitus type 2. World Journal of Diabetes 20156 423-431. (https://doi.org/10.4239/wjd.v6.i3.423)

3 Razak MKA, Akif AM, Nakeeb N \& Rasheed JI. The relationship between mean platelet volume and albuminuria in patients with type 2 diabetes mellitus. Diabetology and Metabolic Syndrome 201913 2633-2639. (https://doi.org/10.1016/j.dsx.2019.07.021) 
4 Athyros VG, Bouloukos VI, Pehlivanidis AN, Papageorgiou AA, Dionysopoulou SG, Symeonidis AN, Petridis DI, Kapousouzi MI, Satsoglou EA, Mikhailidis DP, et al. The prevalence of the metabolic syndrome in Greece: the MetS-Greece Multicentre Study. Diabetes, Obesity and Metabolism 20057 397-405. (https://doi.org/10.1111/ j.1463-1326.2004.00409.x)

5 Pop-Busui R, Boulton AJ, Feldman EL, Bril V, Freeman R, Malik RA, Sosenko JM \& Ziegler D. Diabetic neuropathy: a position statement by the American Diabetes Association. Diabetes Care $2017 \mathbf{4 0}$ 136-154. (https://doi.org/10.2337/dc16-2042)

6 Pop-Busui R, Lu J, Brooks MM, Albert S, Althouse AD, Escobedo J, Green J, Palumbo P, Perkins BA, Whitehouse F, et al. Impact of glycemic control strategies on the progression of diabetic peripheral neuropathy in the bypass angioplasty revascularization investigation 2 diabetes (bari 2D) cohort. Diabetes Care 201336 3208-3215. (https://doi.org/10.2337/dc13-0012)

7 Boulton AJ, Vileikyte L, Ragnarson-Tennvall G \& Apelqvist J. The global burden of diabetic foot disease. Lancet 2005366 1719-1724 (https://doi.org/10.1016/S0140-6736(05)67698-2)

8 Shen J, Liu F, Zeng H, Wang J, Zhao JG, Zhao J, Lu FD \& Jia WP. Vibrating perception threshold and body mass index are associated with abnormal foot plantar pressure in type 2 diabetes outpatients. Diabetes Technology and Therapeutics 201214 1053-1059. (https://doi. org/10.1089/dia.2012.0146)

9 Liu S, Zheng H, Zhu X, Mao F, Zhang S, Shi H, Li Y \& Lu B. Neutrophil-to-lymphocyte ratio is associated with diabetic peripheral neuropathy in type 2 diabetes patients. Diabetes Research and Clinical Practice 2017130 90-97. (https://doi.org/10.1016/j. diabres.2017.05.008)

10 Pop-Busui R, Ang L, Holmes C, Gallagher K \& Feldman EL. Inflammation as a therapeutic target for diabetic neuropathies. Current Diabetes Reports 201616 29. (https://doi.org/10.1007/s11892016-0727-5)

11 Barutta F, Bruno G, Grimaldi S \& Gruden G. Inflammation in diabetic nephropathy: moving toward clinical biomarkers and targets for treatment. Endocrine 201548 730-742. (https://doi.org/10.1007/ s12020-014-0437-1)

12 Zhou J \& Zhou S. Inflammation: therapeutic targets for diabetic neuropathy. Molecular Neurobiology 201449 536-546. (https://doi. org/10.1007/s12035-013-8537-0)

13 Nguyen XM, Lane J, Smith BR \& Nguyen NT. Changes in inflammatory biomarkers across weight classes in a representative US population: a link between obesity and inflammation. Journal of Gastrointestinal Surgery 200913 1205-1212. (https://doi.org/10.1007/ s11605-009-0904-9)

14 Hsieh JY, Smith TD, Meli VS, Tran TN, Botvinick EL \& Liu WF. Differential regulation of macrophage inflammatory activation by fibrin and fibrinogen. Acta Biomaterialia 201747 14-24. (https://doi. org/10.1016/j.actbio.2016.09.024)

15 Streja D, Cressey P \& Rabkin SW. Associations between inflammatory markers, traditional risk factors, and complications in patients with type 2 diabetes mellitus. Journal of Diabetes and its Complications 2003 17 120-127. (https://doi.org/10.1016/s1056-8727(02)00204-0)

16 Don BR \& Kaysen G. Serum albumin: relationship to inflammation and nutrition. Seminars in Dialysis 200417 432-437. (https://doi. org/10.1111/j.0894-0959.2004.17603.x)

17 Li L, Liu B, Lu J, Jiang L, Zhang Y, Shen Y, Wang C \& Jia W. Serum albumin is associated with peripheral nerve function in patients with type 2 diabetes. Endocrine 201550 397-404. (https://doi.org/10.1007/ s12020-015-0588-8)

18 Gonda K, Shibata M, Sato Y, Washio M, Takeshita H, Shigeta H, Ogura M, Oka S \& Sakuramoto S. Elevated neutrophil-to-lymphocyte ratio is associated with nutritional impairment, immune suppression, resistance to S-1 plus cisplatin, and poor prognosis in patients with stage IV gastric cancer. Molecular and Clinical Oncology 20177 1073-1078. (https://doi.org/10.3892/mco.2017.1438)

19 Unal D, Orhan O, Eroglu C \& Kaplan B. Prealbumin is a more sensitive marker than albumin to assess the nutritional status in patients undergoing radiotherapy for head and neck cancer. Contemporary Oncology 201317 276-280. (https://doi.org/10.5114/wo.2013.35281)

20 Hrnciarikova D, Juraskova B, Hyspler R, Solichova D, Ticha A, Klemera P, Hronek M \& Zadak Z. A changed view of serum prealbumin in the elderly: prealbumin values influenced by concomitant inflammation. Biomedical Papers of the Medical Faculty of the University Palacky, Olomouc, Czechoslovakia $2007151273-276$. (https://doi.org/10.5507/bp.2007.046)

21 Myron Johnson A, Merlini G, Sheldon J, Ichihara K \& Scientific Division Committee on Plasma Proteins (C-PP). Clinical indications for plasma protein assays: transthyretin (prealbumin) in inflammation and malnutrition. Clinical Chemistry and Laboratory Medicine 200745 419-426. (https://doi.org/10.1515/ CCLM.2007.051)

22 Dennis RA, Johnson LE, Roberson PK, Heif M, Bopp MM, Cook J \& Sullivan DH. Changes in prealbumin, nutrient intake, and systemic inflammation in elderly recuperative care patients. Journal of the American Geriatrics Society 200856 1270-1275. (https://doi. org/10.1111/j.1532-5415.2008.01789.x)

23 Yue W, Liu Y, Ding W, Jiang W, Huang J, Zhang J \& Liu J. The predictive value of the prealbumin-to-fibrinogen ratio in patients with acute pancreatitis. International Journal of Clinical Practice 2015 69 1121-1128. (https://doi.org/10.1111/ijcp.12682)

24 Tang S, Lin L, Cheng J, Zhao J, Xuan Q, Shao J, Zhou Y \& Zhang Y. The prognostic value of preoperative fibrinogen-to-prealbumin ratio and a novel FFC score in patients with resectable gastric cancer. BMC Cancer 202020 382. (https://doi.org/10.1186/s12885-020-06866-6)

25 American Diabetes Association. 2. Classification and diagnosis of diabetes. Diabetes Care 201639 (Supplement 1) S13-S22. (https://doi. org/10.2337/dc16-S005)

26 Tesfaye S, Boulton AJ, Dyck PJ, Freeman R, Horowitz M, Kempler P, Lauria G, Malik RA, Spallone V, Vinik A, et al. Diabetic neuropathies update on definitions, diagnostic criteria, estimation of severity, and treatments. Diabetes Care 201033 2285-2293. (https://doi. org/10.2337/dc10-1303)

27 Fibrinogen Studies Collaboration, Danesh J, Lewington S, Thompson SG, Lowe GD, Collins R, Kostis JB, Wilson AC, Folsom AR, Wu K, et al. Plasma fibrinogen level and the risk of major cardiovascular diseases and nonvascular mortality: an individual participant meta-analysis. JAMA 2005294 1799-1809. (https://doi. org/10.1001/jama.294.14.1799)

28 Kotbi S, Mjabber A, Chadli A, El Hammiri A, El Aziz S, Oukkache B, Mifdal H, Nourichafi N, Kamal N, Habbal R, et al. Correlation between the plasma fibrinogen concentration and coronary heart disease severity in Moroccan patients with type 2 diabetes. Prospective study. Annales d'Endocrinologie 201677 606-614. (https:// doi.org/10.1016/j.ando.2015.02.004)

29 Zhang J, Wang Y, Zhang R, Li H, Han Q, Wu Y, Wang S, Guo R, Wang T, Li L, et al. Serum fibrinogen predicts diabetic ESRD in patients with type 2 diabetes mellitus. Diabetes Research and Clinical Practice 2018141 1-9. (https://doi.org/10.1016/j. diabres.2018.04.025)

30 Arık HO, Yalcin AD, Gumuslu S, Genç GE, Turan A \& Sanlioglu AD. Association of circulating sTRAIL and high-sensitivity CRP with type 2 diabetic nephropathy and foot ulcers. Medical Science Monitor 2013 19 712-715. (https://doi.org/10.12659/MSM.889514)

Received in final form 30 July 2020

Accepted 6 August 2020

Accepted Manuscript published online 7 August 2020 